\title{
In situ tissue engineering for tracheal reconstruction using a luminar remodeling type of artificial trachea
}

\author{
Tatsuo Nakamura, MD, ${ }^{\mathrm{a}}$ Toshihiko Sato, ${ }^{\mathrm{a}}$ Masato Araki, MD, ${ }^{\mathrm{a}}$ Satoshi Ichihara, MD, ${ }^{\mathrm{a}}$ Akira Nakada, MD, ${ }^{\mathrm{a}}$ \\ Makoto Yoshitani, MD, ${ }^{\mathrm{a}}$ Shin-ichi Itoi, MD, ${ }^{\mathrm{a}}$ Masaru Yamashita, MD, ${ }^{\mathrm{b}}$ Shin-ichi Kanemaru, MD, \\ Kouichi Omori, MD, ${ }^{\mathrm{c}}$ Yoshio Hori, MD, ${ }^{\mathrm{a}}$ Katsuaki Endo, MD, ${ }^{\mathrm{d}}$ Yuji Inada, MD, ${ }^{\mathrm{a}}$ and \\ Katsumi Hayakawa, MD ${ }^{\mathrm{a}}$
}

Background: After successful trials of tracheal reconstruction using mesh-type prostheses in canine models, the technique has been applied clinically to human patients since 2002. To enhance tissue regeneration, we have applied a new tissue engineering approach to this mesh-type prosthesis.

\begin{abstract}
Methods: The prosthesis consists of a polypropylene mesh tube reinforced with a polypropylene spiral and atelocollagen layer. The cervical tracheas of 18 beagle dogs were replaced with the prosthesis. The collagen layer was soaked with peripheral blood in 6 of the dogs, with bone marrow aspirate in another 6 , and with autologous multipotential bone marrow-derived cells (mesenchymal stem cells) in another 6 . The dogs were humanely killed at 1 to 12 months after the operation.

Results: All 18 dogs survived the postoperative period. Bronchoscopically, 3 of 4 dogs in the peripheral blood group showed stenosis, whereas no stenosis was evident in all 8 of the dogs in the bone marrow and mesenchymal stem cell groups 6 months after the operation. Faster epithelialization and fewer complications, such as mesh exposure and luminal stenosis, were observed in these two groups than in the peripheral blood group. Histologically, the cells from autologous bone marrow were found to proliferate into the tracheal tissue during the first month. Cilial movement in these two groups was faster than that in the peripheral blood group and recovered to $80 \%$ to $90 \%$ of the normal level.
\end{abstract}

Conclusions: Bone marrow aspirate and mesenchymal stem cells enhance the regeneration of the tracheal mucosa on this prosthesis. This in situ tissue engineering approach may facilitate tracheal reconstruction in the clinical setting.

End-to-end anastomosis has been the standard clinical method for tracheal reconstruction since the 1970s. ${ }^{1}$ However, in cases in which the resected segment is more than $6 \mathrm{~cm}$ long, high tension at the anastomosis site can sometimes cause severe and fatal postoperative complications such as dehiscence. ${ }^{2}$ A variety of artificial tracheas have been designed for such cases and assessed, but so far none has proved satisfactory for clinical use.

We have developed a new mesh-type tracheal prosthesis on which autologous tracheal tissue is encouraged to regenerate on a collagen scaffold. We have already demonstrated that this prosthesis can be applied safely for circumferential

\footnotetext{
From the Department of Bioartificial Organs, ${ }^{\text {a }}$ Institute for Frontier Medical Sciences, Kyoto University, the Department of Otolaryngology, ${ }^{b}$ Kyoto University, Kyoto, Japan; the Department of Otolaryngology, ${ }^{\mathrm{c}}$ Fukushima Medical University, Fukushima, Japan; and the Department of Physiology, ${ }^{\mathrm{d}}$ Kyoto Nerve Regeneration Research Center, Kyoto, Japan.

This work was supported partly by a Health and Labor Science Research Grant for Research on the Human Genome, and Tissue Engineering, from the Ministry of Health, Labor and Welfare of Japan.

Received for publication Feb 2, 2008; revisions received May 12, 2008; accepted for publication July 3, 2008; available ahead of print May 13, 2009.

Address for reprints: Tatsuo Nakamura, MD, Department of Bioartificial Organs, Institute for Frontier Medical Sciences, Kyoto University, 53 Kawahara cho, Sakyoku, Kyoto 606-8507, Japan (E-mail: nakamura@frontier.kyoto-u.ac.jp).

J Thorac Cardiovasc Surg 2009;138:811-9

$0022-5223 / \$ 36.00$

Copyright (c) 2009 by The American Association for Thoracic Surgery

doi:10.1016/j.jtcvs.2008.07.072
}

replacement of the cervical trachea, ${ }^{3}$ thoracic trachea, ${ }^{2,4}$ or carinal bifurcation ${ }^{5}$ in canine models. On the basis of the success of these experimental animal studies, clinical application of the tracheal prosthesis was begun for human patients with tracheal defects in $2002 .^{6}$

In animal experiments, the initial type of prosthesis that was simply coated with amorphous collagen often developed mesh exposure or ulcers on the inner mucosa. ${ }^{7}$ This indicated that early epithelial regeneration was a key factor required for successful use of this mesh-type tracheal prosthesis. In other words, enhancement of tissue recovery on the prosthesis was a crucial requirement for tracheal reconstruction.

In clinical trials, the prosthesis was moistened with autologous peripheral blood before anastomosis. ${ }^{6}$ If tissue regeneration can be facilitated with an improved method, it would certainly contribute to the clinical feasibility of a tracheal prosthesis. Therefore, in the present study, we also used bone marrow aspirate and a suspension of bone marrow-derived mesenchymal stem cells (MSCs) as alternatives to peripheral blood. We also determined whether the seeded autologous bone marrow cells proliferated into the tracheal tissue in situ.

\section{MATERIALS AND METHODS Collagen}

The collagen used was extracted from skin of young pigs (6 months old weighing about $120 \mathrm{~kg}$ ) (supplied by Nippon Meatpackers Inc, Ibaraki, Japan). The extracted collagen was then digested with pepsin at $4^{\circ} \mathrm{C}$ for 


\section{Abbreviations and Acronyms \\ $\mathrm{CBF}=$ cilial beat frequency \\ MRI $=$ magnetic resonance imaging \\ MSC $=$ mesenchymal stem cell}

48 hours. The supernatant consisted of type 1 collagen $(70 \%-80 \%)$, and the rest was type 3 collagen, as confirmed by sodium dodecyl sulfatepolyacrylamide gel electrophoresis. During the pepsin treatment, the telopeptide of collagen, which is thought to have antigenicity, was removed, leaving only the atelocollagen.

\section{Prosthesis}

A straight tube $16 \mathrm{~mm}$ in diameter was manufactured from a sheet of polypropylene mesh with a pore size of $260 \times 10^{-6} \mathrm{~m}$ (Bard mesh; Davol Inc, Cranston, RI) by melting the edges together. The tube was then reinforced with a polypropylene monofilament yarn $(0.8 \mathrm{~mm}$ in diameter) (Figure 1$)$. The yarn was spirally attached to the external surface of the mesh tube by thermal melt-bonding at 5-mm intervals and further fixed with 7-0 Prolene polypropylene suture (Ethicon, Inc, Somerville, NJ). The polypropylene spiral provided the tube with stiffness against compression. The interval of the yarn was determined so that the mechanical resistance against compression was the same as that of the native trachea. ${ }^{8}$

This polypropylene tube was then exposed to a coronal discharge at $9 \mathrm{kV}$ for 10 minutes to activate the surface. The prosthesis was subsequently coated 10 times with the $1 \%$ collagen solution. The activated prosthesis was covalently immobilized with collagen molecules and then further physically coated with collagen to promote host tissue incorporation and render the prosthesis airtight during the initial stage of implantation. This coating process was repeated 20 times.

The collagen-coated prosthesis was placed in a cylindrical Teflon mold with an inner diameter of $37 \mathrm{~mm}$ and a depth of $6 \mathrm{~cm}$. At the same time, a 5-mm-diameter Teflon tube was inserted into the center of the prosthesis. The $1 \%$ collagen solution, which had been stirred at $8000 \mathrm{rpm}$ for 15 minutes, was poured into the space between the outer mold and the inner tube and then freeze-dried. In this freeze-drying process, the cast collagen became a porous structure with a pore size range of 100 to $500 \times 10^{-6} \mathrm{~m}$. Finally, the prosthesis was subjected to dehydrothermal treatment $140^{\circ} \mathrm{C}$ in vacuo for 24 hours to induce cross-links between the collagen molecules to prevent early breakdown of the collagen in vivo.

\section{Animal Experiments}

Eighteen beagle dogs weighing between 8 and $14 \mathrm{~kg}$ were divided at random into three groups. The cervical trachea was cut transversely 20 $\mathrm{mm}$ caudal to the thyroid cartilage, and a tracheal segment $(40-50 \mathrm{~mm})$ including seven tracheal cartilages was removed with the animal under general anesthesia. During the operation, the prosthesis was preclotted with $5 \mathrm{~mL}$ of autologous peripheral blood in 8 of the dogs (peripheral blood group). In another $8 \mathrm{dogs}$, the prosthesis was soaked with $5 \mathrm{~mL}$ of bone marrow aspirate obtained from the femoral bone during the operation (bone marrow group). In the remaining $8 \mathrm{dogs}$, from which bone marrow aspirate had been taken and incubated in advance, the prosthesis was soaked with an incubated bone marrow cell suspension (MSC group). In these 8 dogs of the MSC group, $2 \mathrm{~mL}$ of bone marrow aspirate was taken from the femur 4 weeks before the operation. The bone marrow aspirate was incubated with Dulbecco modified Eagle medium containing $10 \%$ fetal bovine serum. The incubation medium was changed every 3 days from the fifth day. Floating cells were removed when the medium was changed. Some cells became attached to the bottom of the incubation bottle and proliferated. These proliferated cells were detached with trypsin, and a suspension of these cells $\left(10^{7}\right)$ was used in the operating room.

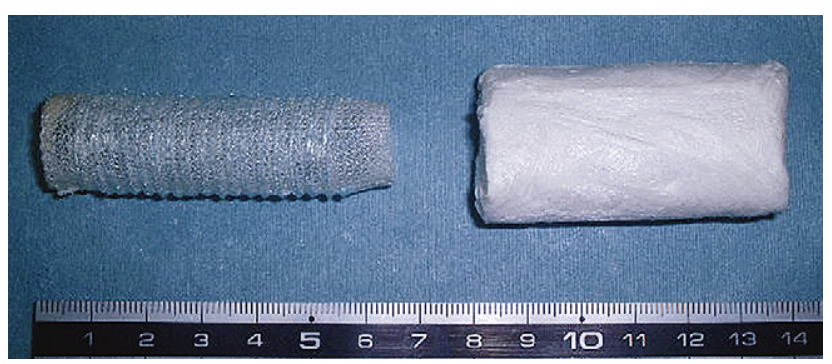

FIGURE 1. Mesh-type tracheal prosthesis. The framework is a polypropylene mesh tube reinforced with a spiral stent (right). Freeze-dried collagen layers were made both inside and outside the framework as a scaffold of tissue regeneration (left).

The porous collagen layer inside and outside the lumen shrank in volume on soaking. The prosthesis and the cut ends of the trachea were anastomosed with 3-0 Vicryl (Ethicon) interrupted sutures, the cut ends of the tracheal stumps being inserted into the prosthesis lumen (Figure 2). Neither omentopexy nor fibrin glue was used.

A 1000-mg dose of ampicillin was injected intramuscularly on the day of the operation, and a 500-mg dose was administered orally every day for 1 month thereafter. Bronchial cleaning with a fiberscope was only performed immediately after the operation on the same day. No additional care other than routine management was provided for the animals.

\section{Bronchoscopic Examinations and Cilial Beat Frequency (CBF) Examination}

A bronchofiberoscope (model BF1T20; Olympus Optical Co Ltd, Tokyo, Japan) examination was performed periodically under general anesthesia with an intramuscular injection of ketamine hydrochloride $(10 \mathrm{mg} / \mathrm{kg})$
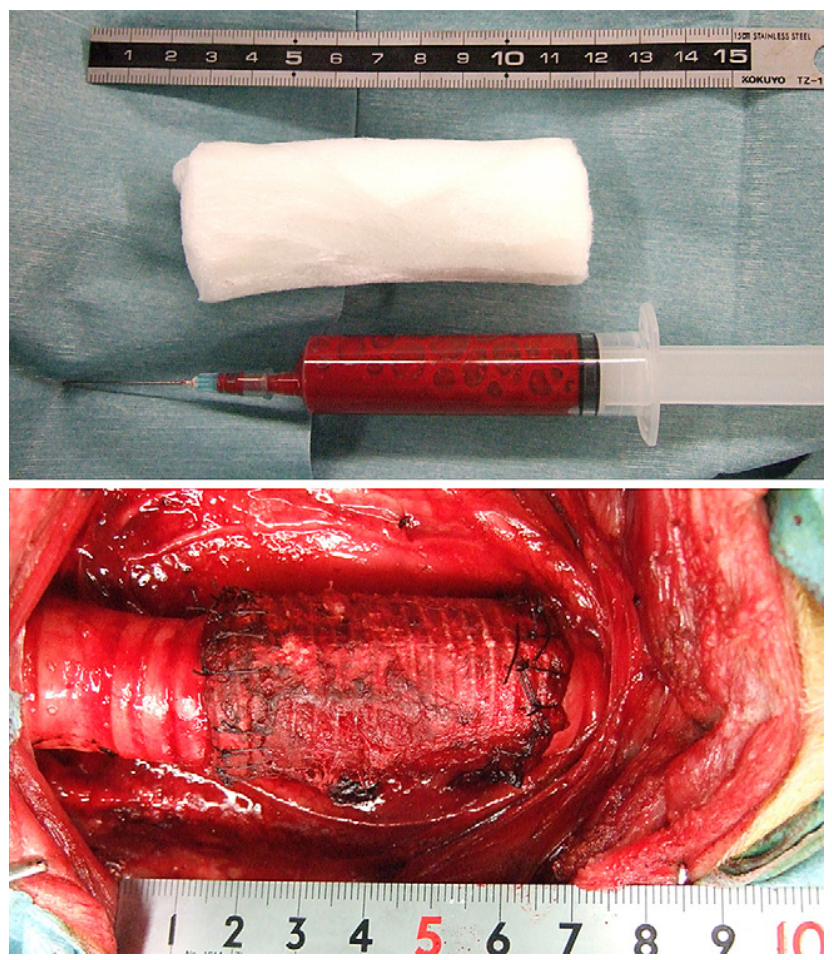

FIGURE 2. Intraoperative view of the reconstruction of a canine trachea with the prosthesis. The prosthesis was soaked with each medium before anastomosis. The prosthetic wall became airtight with this process. 
TABLE 1. Results of tracheal reconstruction with prosthesis

\begin{tabular}{|c|c|c|c|c|c|}
\hline Dog no. & Soak medium & Observation (mo) & Incorporation to host & Stenosis & Erosion \\
\hline 1 & Peripheral blood & 1 & + & - & - \\
\hline 2 & Peripheral blood & 3 & + & - & - \\
\hline 3 & Peripheral blood & 6 & + & - & - \\
\hline 4 & Peripheral blood & 6 & + & + & - \\
\hline 5 & Peripheral blood & 12 & + & + & + \\
\hline 6 & Peripheral blood & 12 & + & + & - \\
\hline 7 & Bone marrow aspirate & 1 & + & - & - \\
\hline 8 & Bone marrow aspirate & 3 & + & - & - \\
\hline 9 & Bone marrow aspirate & 6 & + & - & - \\
\hline 10 & Bone marrow aspirate & 6 & + & - & - \\
\hline 11 & Bone marrow aspirate & 12 & + & - & - \\
\hline 12 & Bone marrow aspirate & 12 & + & + & - \\
\hline 13 & MSC suspension* & 1 & + & - & - \\
\hline 14 & MSC suspension & 3 & + & - & - \\
\hline 15 & MSC suspension & 6 & + & - & - \\
\hline 16 & MSC suspension & 6 & + & - & - \\
\hline 17 & MSC suspension & 12 & + & - & - \\
\hline 18 & MSC suspension & 12 & + & + & - \\
\hline
\end{tabular}

Mesenchymal stem cells (MSC) were labeled with fluorescent marker DiI. *Stenosis is defined as a reduction of the tracheal lumen by more than one third.

and xylazine hydrochloride $(4 \mathrm{mg} / \mathrm{kg})$ to examine the luminal surface of the prosthesis. The replaced segments were examined carefully. Mesh exposure exceeding one third of the circumference was define as " + ", and less than one third as " +." Stenosis was defined as a reduction of more than one third of inner luminal area.

At 6 months and 12 months, samples of the tracheal epithelium were removed with a brush inserted through the channel of the bronchoscope to evaluate cilial movement. The brush, which scrubbed the target (reconstructed) portion of the tracheal mucosa, was washed with culture medium. This medium (containing the scrubbed tracheal mucosa cells) dropped on a glass slide was then observed with a light microscope. The movement of the cilia on the slide was counted at $37^{\circ} \mathrm{C}$ with a photomultiplier system ${ }^{9}$ connected to the microscope.

Cilial movement frequency was compared among the three groups by analysis of variance and a $t$ test. All frequency measurements obtained are expressed as the mean ( \pm standard deviation) of at least 10 determinations.

\section{Magnetic Resonance Imaging}

Magnetic resonance imaging (MRI) of the reconstructed trachea and adjacent organs was conducted on $1 \mathrm{dog}$ from each group 12 months after implantation. The MRI system used was a Sonata MRI System with a static magnetic flux density of $1.5 \mathrm{~T}$ (Siemens Medical System, Erlangen, Germany) using a phase-array body coil with four active segments. The dog was anesthetized with an intravenous injection of propofol (Diprivan; $5 \mathrm{mg} \cdot \mathrm{min}^{-1} \cdot \mathrm{kg}^{-1}$ ) during the imaging.

\section{Histologic Examination}

One dog in each group was humanely killed with an injected overdose of sodium pentobarbital 1 and 3 months after tracheal reconstruction. Two dogs in each group were humanely killed 6 months after tracheal reconstruction. At 12 months, 2 dogs in the peripheral blood group and 1 each in the bone marrow and MSC groups were humanely killed. The remaining dog in each of the bone marrow and MSC groups was allowed to survive for further observation.

En bloc resection of the prosthesis with the native trachea was carried out for histopathologic evaluation. The epithelial cells on the inner surface were checked with a scanning electron microscope (S-450; Hitachi Ltd, Tokyo, Japan).

All of the surgical and euthanasia procedures were performed in accordance with the "Guide for the Care and Use of Laboratory Animals" pub- lished by the National Institutes of Health (NIH Publication No. 85-23, revised 1985). The experimental protocol was approved by Animal Experimental Committee of Kyoto University.

\section{RESULTS}

All animals in the three groups had uneventful postoperative courses, and no symptoms that necessitated unscheduled humane killing of an animal occurred (Table 1).

\section{Bronchoscopic Examination}

Bronchoscopic examinations at 1 month revealed that the luminal surface of the implanted prosthesis appeared glossy and whitish, suggesting complete coverage of the lumen by regenerated tissue (Figure 3). No mesh exposure was recognized even in the middle of the prosthesis, except in $1 \mathrm{dog}$ (Table 1, dog 5), in which an area of exposed mesh measuring about $5 \times 2 \mathrm{~mm}$ was observed at the center of the prosthesis at 1 month. Despite this mesh exposure, the dog was asymptomatic and its general condition was good, and no retention of sputum was observed in the lumen of the prosthesis up to 12 months. Stenosis of the prosthetic lumen was observed in 3 of the dogs in the peripheral blood group and in $1 \mathrm{dog}$ in each of the other two groups. This stenosis occurred in the middle of the prosthesis and not at the sites of anastomosis. The definition of stenosis used in this study was reduction of the tracheal luminal diameter by more than one third. Whereas both dogs in the bone marrow group and the MSC group had only mild stenosis, severe stenosis that reduced the diameter of the tracheal lumen by more than half occurred in 3 of the 4 dogs in the peripheral blood group. The narrowest luminal diameter was $9 \mathrm{~mm}$ in both the bone marrow group and the MSC group, whereas that in the peripheral blood group was $6 \mathrm{~mm}$. 

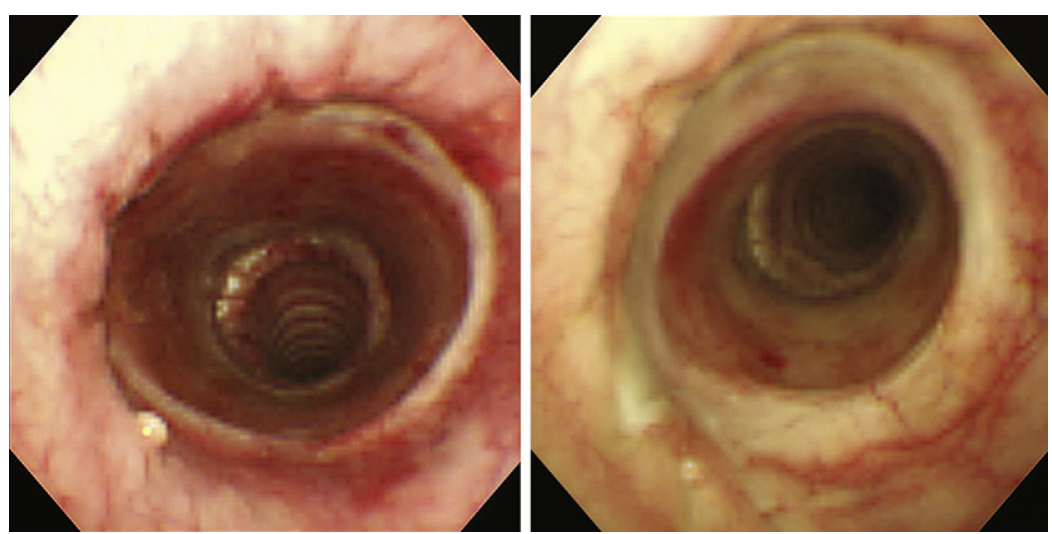

FIGURE 3. Bronchoscopic views of the reconstructed site of the bone marrow group (dog 11) 1 month (left) and 12 months (right) after the operation. Neither stenosis nor granulation is evident at the site of anastomosis with the trachea. Complete coverage of the mesh by regenerated tissue is evident.

\section{Macroscopic Examination}

At 1 month the inner surface of the prosthesis was covered with glossy tissue in all three groups (Figure 4). The prostheses were incorporated into the native tracheas, and there were no major complications such as erosion of the surrounding organs, abscess formation, pneumothorax, anastomotic dehiscence, or prosthetic dislocation in any of the 18 dogs that were humanely killed on schedule (Table 1).

\section{MRI}

MRI at 12 months indicated no scar formation around the prosthesis, and the inner lumen of the tube was maintained in the cervical space in all three groups (Figure 5). The implanted prostheses were well incorporated with the native trachea at the interfaces, and there was no morbid tissue reaction between adjacent organs including major blood vessels.

\section{Histologic Examinations}

Examination with a light microscope at 1 month revealed that the polypropylene mesh and polypropylene spiral had become buried in newly regenerated connective tissue and that the implanted collagen had disappeared. Capillary ingrowth was observed in the regenerated tracheal tissue in all dogs of the three groups. After 6 months, formation of respiratory epithelium was confirmed histologically from the upper to the lower anastomotic site of the prosthesis (Figure 6).

Although the extent of the epithelial lining of the regenerated mucosa on the prosthesis varied, 9 of the 12 dogs in the bone marrow and MSC groups had an epithelial lining covering more than $50 \%$ of the length of the prosthesis. In contrast, after 3 and 6 months, the peripheral blood group had poor epithelial lining, in which the squamous epithelium was thicker than that of the columnar bronchial epithelium. After 12 months, the extent of the epithelial lining in the peripheral blood group was similar to that in the other two groups. In each animal, ciliated columnar epithelium was observed near the anastomoses, the proportion of nonciliated cuboidal epithelium increased with distance from the anastomoses, and squamous epithelium was observed in the center of the prosthesis (Figure 7).

An area of exposed mesh measuring $5 \times 2 \mathrm{~mm}$ without any sign of local infection was observed in $\operatorname{dog} 5$ of the peripheral blood group. No granulation tissue formation was detected in the lumen.

Histologic examination of the stenotic part of the prosthesis in 5 dogs showed that the connective tissue, including vessels, had invaded the mesh pores, covering the inner surface of the prosthesis. Also in these animals, the neoepithelium appeared stretched from the cut end of the tracheal stump over the connective tissue layer.
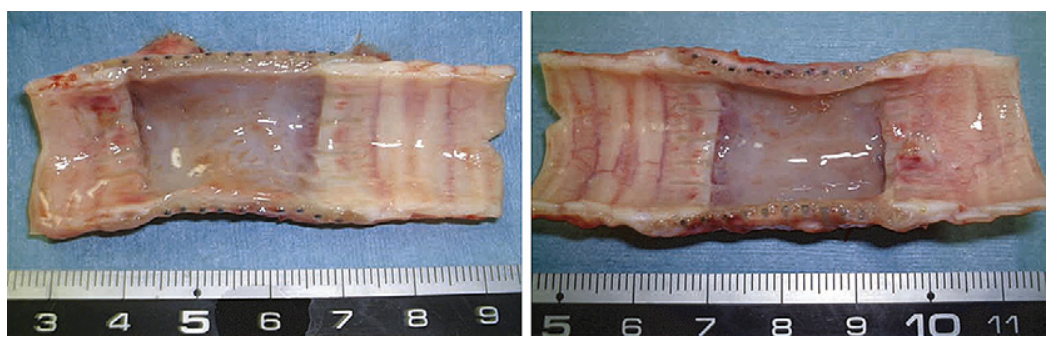

FIGURE 4. Luminal surface of the prosthesis (bone marrow group, $\operatorname{dog} 8$ ). Three months after reconstruction, the prosthesis was incorporated into the native tracheas and inner surface was covered with glossy and whitish tissue. Polypropylene stents were seen in the cross-section of the reconstructed tracheal wall. 


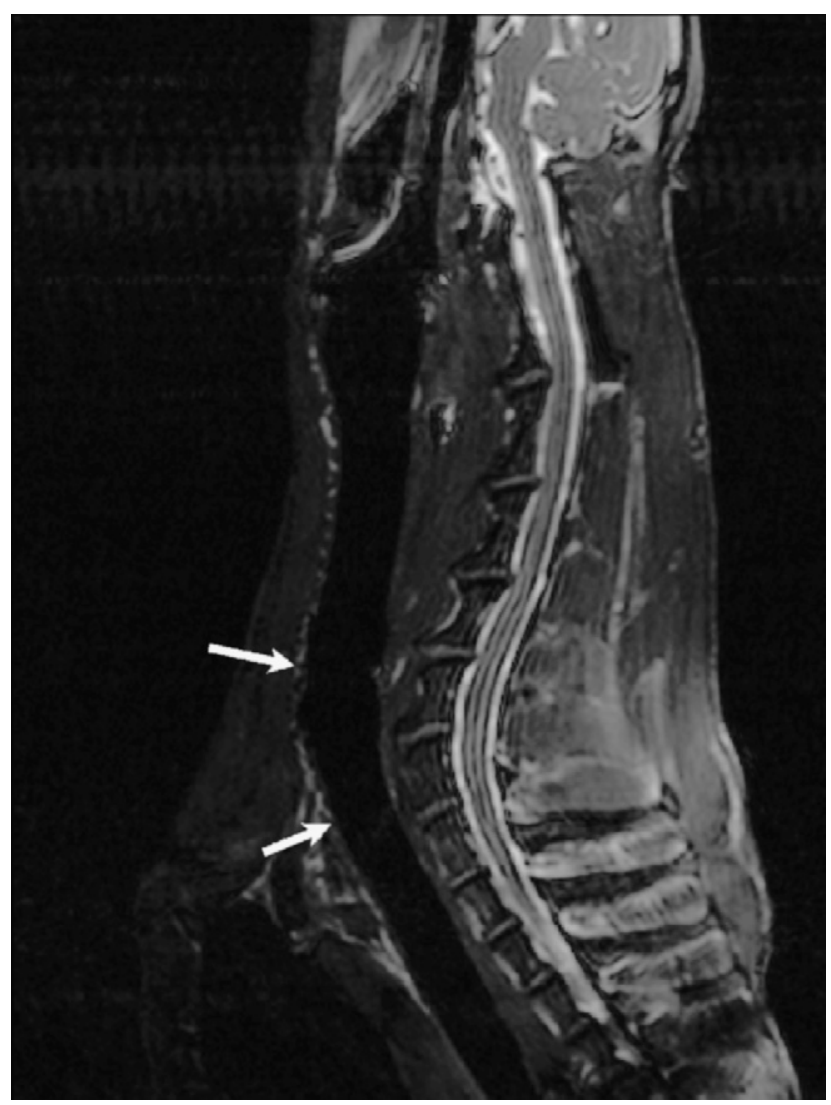

FIGURE 5. MRI appearance of the prosthesis implanted into the cervical trachea of a dog 12 months after the operation (MSC group). Replaced area is indicated by the arrows.

Scanning electron microscopy examination revealed that the regenerated epithelial cells near the anastomoses possessed cilia similar to those of the normal tracheal epithelium in all groups. Moreover, a few ciliated epithelial cells were observed in areas where only a squamous cell lining was demonstrated by light microscopy (Figure 7).

Observation by transmission electron microscopy showed long and uniform cilia on the luminal surface over a large area, gathering closely together (Figure 8) on the regenerated part of the trachea in all three groups. In the area where ciliated cells were absent, the luminal surface was covered with the squamous cells.

\section{Comparison of CBF in the Regenerated Mucosa}

$\mathrm{CBF}$ in the regenerated tracheal mucosa is shown in Figure 9. CBF of the same portion of the trachea measured in intact normal beagle dogs using the same system was $14.6 \pm 3.5$.

At 6 months, CBF values in the bone marrow group and MSC group had recovered to almost the normal range and were significantly higher than that in the control group. There were no significant differences in CBF among the three groups at 12 months $(P>.05)$.

\section{$\mathbf{L}$}

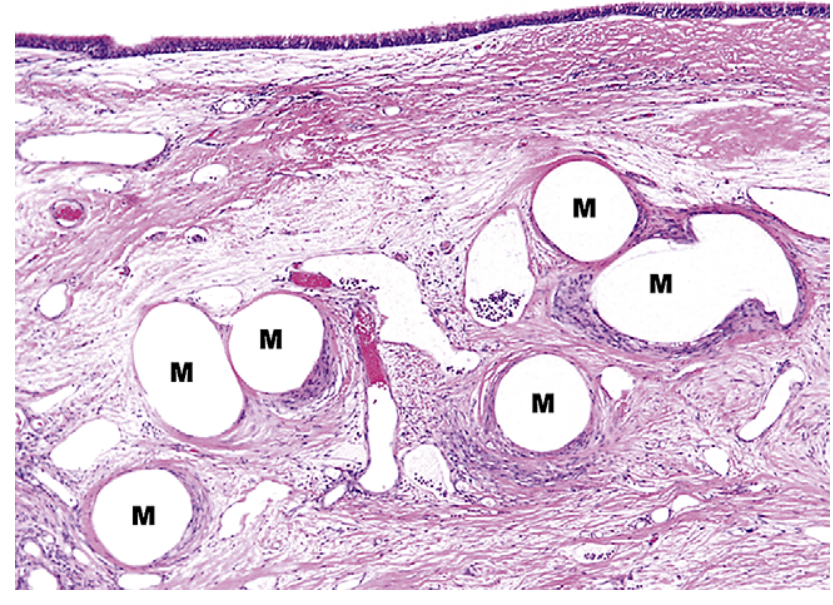

FIGURE 6. Regenerated mucosa with epithelial lining on the mesh of the prosthesis from a dog in the bone marrow group after 12 months. The mucosa is thicker than the normal tracheal mucosa. $L$, Tracheal lumen; $M$, mesh of the prosthesis (hematoxylin and eosin staining; original magnification, $\times 400)$.

\section{Proliferation of Bone Marrow-Derived Cells}

To determine the fate of the MSCs, we used MSC labeled with Di-I (Cell Tracer CM-DiI; Molecular Probes, Leiden, The Netherlands) in 1 dog (no. 13) of the MSC group, which was killed 1 month after the operation. At this time, the DiI-labeled MSCs were recognized in the regenerated tracheal tissue. They remained in the submucosal tissue around the polypropylene mesh and were not present in the mucosa (Figure 10).

\section{DISCUSSION}

Direct end-to-end anastomosis has been the "gold standard" for airway reconstruction. ${ }^{10}$ However, the range of possible reconstruction with this method is limited, and even within the feasible range (less than $6 \mathrm{~cm}$ ), tensionreducing procedures are required during the operation. In the case of resections approaching the theoretical maximum, patients must endure a stressful drawn-in chin position with neck fixation for at least 2 weeks after the operation to avoid concentrating tension on the sites of anastomosis. ${ }^{2}$ Thus, direct apposition and suturing of the tracheal stumps tends to place a heavy burden on the patient. For resolution of such problems, the use of a tracheal substitute makes reconstruction easier, and as a result, the surgical approach can be expanded with the use of a tracheal prosthesis.

Many types of material have been examined for use in tracheal prostheses since the first report by Daniel ${ }^{11}$ in 1948. A prominent milestone in the history of artificial trachea development was the introduction of a silicone rubber prosthesis in the 1960s, which became commercially available in the $1970 \mathrm{~s}^{12}$ and was used clinically for a short time. However, because re-epithelialization could not be expected on the 

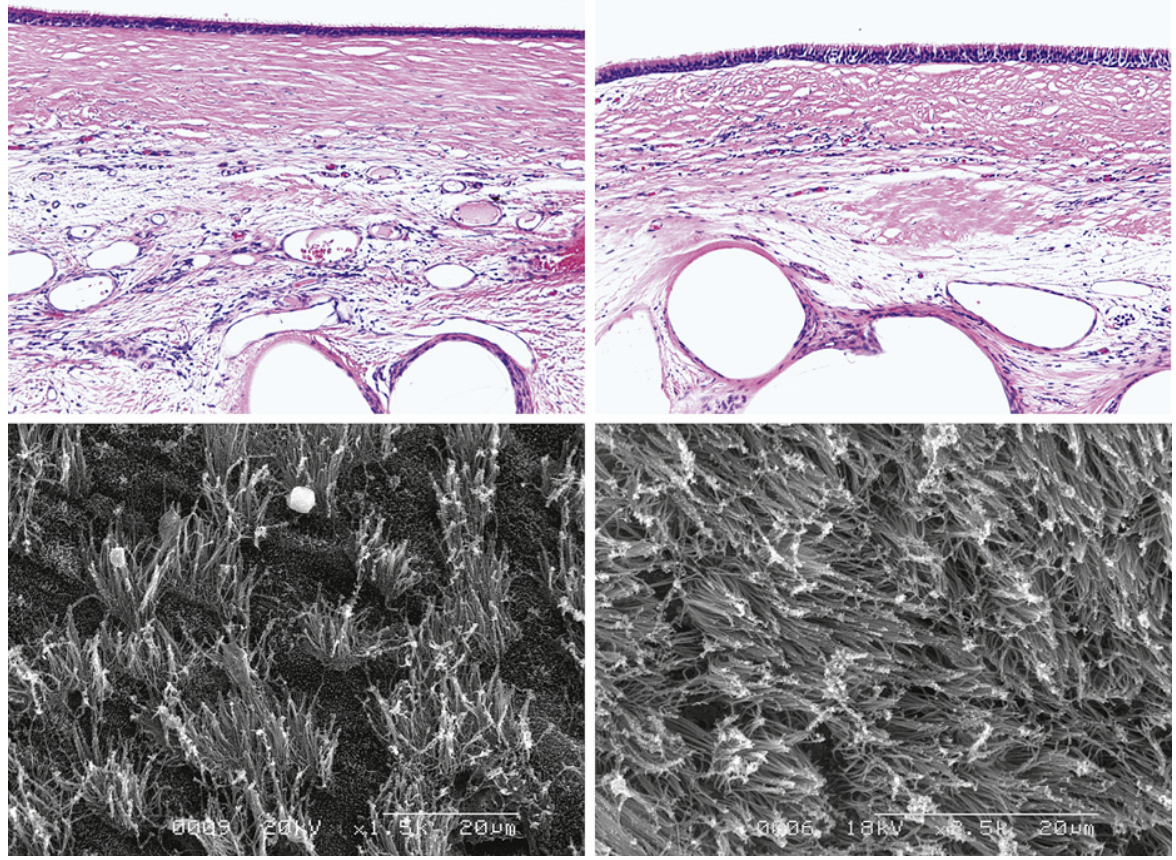

FIGURE 7. Ciliated epithelium is present on the regenerated mucosa near the anastomosis (upper left), but only squamous epithelium is present in the middle of the prosthesis (upper right) in a dog in the bone marrow group after 12 months (hematoxylin and eosin staining; original magnification, $\times 200$ ). Bottom, Scanning electron microscopy (SEM) views of the luminal surface. Dense cilia like those of the normal tracheal epithelium are present on the prosthesis near the anastomosis (lower left) and a few ciliated epithelial cells are present in areas where only a squamous cell lining was demonstrated by light microscopy (lower right). Although these findings were evident in all three groups, there were more ciliated cells in the bone marrow and MSC groups than in the peripheral blood group.

inner lumen, formation of granulation tissue or dehiscence occurred at the interface between the prosthesis and the native trachea, usually within several months. ${ }^{13}$ For this reason, the nonporous tracheal prosthesis is now seldom used clinically.

One of the reasons that prosthetic tracheal reconstruction is more difficult than use of a vascular prosthesis may be that the trachea is in contact with the external environment (ie, it is an airway). At the interface of an implanted foreign body and host tissue, the wound healing process continues until the implanted foreign body is completely encapsulated or is rejected from the implant site. Such phenomena are often observed clinically by surgeons in relation to suture materials used on skin incisions. In this respect, an artificial trachea contrasts conspicuously with an artificial vascular graft, which has no external interface after implantation.

With this in mind, we $\mathrm{e}^{3,4,7,8,14}$ have designed a series of mesh-type tracheal prostheses that eliminate the interface facing the airway. In such prostheses, autologous tissue invades the pore spaces, and the interface of the foreign material with the airway finally disappears with reepithelialization of the inner lumen. ${ }^{15}$

However, one problem with the mesh-type prosthesis that needs to be overcome is insufficient air sealing of the mesh structure, especially just after implantation. Indeed, to seal the porous tracheal prosthesis, a 2-stage operation was once designed for ensuring that the prosthesis was airtight. ${ }^{16,17}$ However such a 2-stage operation places a heavy burden on the patient and seems too complicated for clinical use.

Therefore, to seal the mesh, we ${ }^{14}$ began to apply collagen in the early 1980s in the expectation that the collagen might act not only as a palliative sealant but also as a scaffold for tissue regeneration. Collagen, the main element of the extracellular matrix, has been reported to play several important roles in tissues, such as cell adhesion, detachment, transformation, and proliferation. ${ }^{18}$ For example, type 1 collagen is reported to promote the differentiation of tracheal ciliated epithelium. $^{19}$

In this connection, tissue engineering, whereby a variety of tissues can be fabricated in incubation rooms using cell culture techniques, has been a focus of attention since $1990 .^{20}$ Tissue engineering has also been applied for tracheal reconstruction. In 1994, epithelial tissue was first induced on a tissue-engineered trachea. ${ }^{21}$ In that study, tracheal epithelial cells were isolated from newborn lambs in advance to induce an epithelial lining on the reconstructed site. These epithelial cells were injected into a previously manufactured cylindrical cartilage tube made from cells harvested from calves. These in vitro-formed tubes were then implanted into subcutaneous pockets of 10 nude mice, aged 6 weeks. Four of the 10 implanted tubes were found to develop an epithelial lining on the inner surface, whereas 

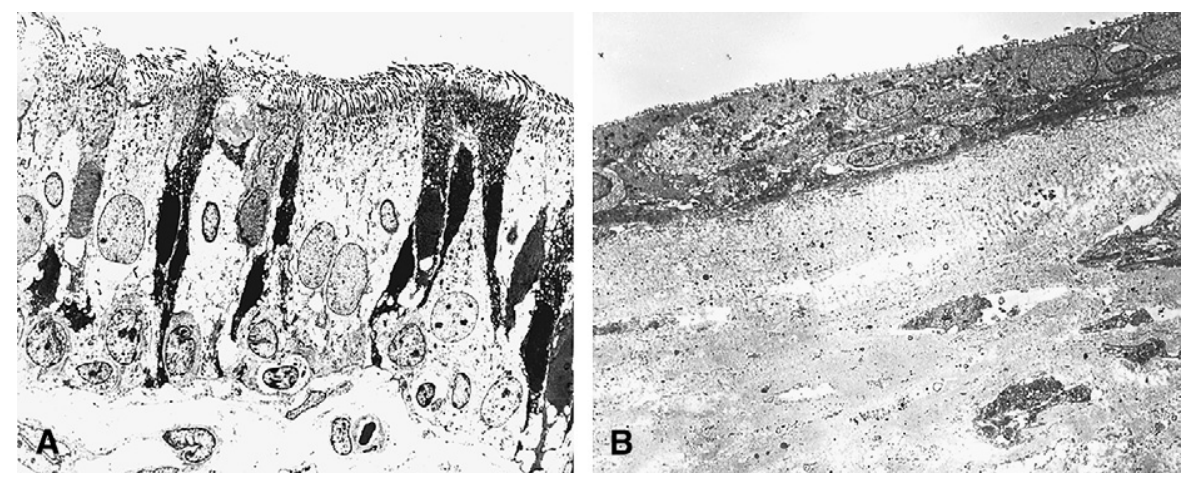

FIGURE 8. Transmission electron microscopic view of the regenerated mucosa of the reconstructed segment (center) 12 months after reconstruction in the MSC group (A). In the area where ciliated cells were absent, the luminal surface was covered with the squamous cells (B).

the other 6 tubes became infected. In 2001, in vitro-cultured tracheal epithelial cells were successfully transplanted onto a prefabricated capsule pouch surface with fibrin glue for possible use in tracheal reconstruction. ${ }^{22}$

Development of tracheal cartilage has also been studied in rats by means of tissue engineering. ${ }^{23}$ Kojima and coworkers $^{24}$ have successfully constructed an autologous tissue-engineered trachea with sheep nasal chondrocytes and used it to reconstruct the trachea of 6 sheep. However, because of malacia and stenosis, their animals survived only 2 to 7 days after surgery. Okamoto and coworkers ${ }^{25}$ have made tracheal cartilage using bone morphogenetic protein 2 in dogs. In their canine model, a $1 \times 5-\mathrm{cm}$ slit defect of the tracheal cartilage was created in the trachea in advance, while carefully preserving the tracheal mucosa. Subsequently, regeneration of the cartilage was recognized around the stumps of the resected cartilages. Although these tissue engineering trials have met with some limited success, achievement of a long-term durable tracheal cartilage with mechanical properties similar to those of native tracheal cartilage still seems a long way off.

In situ tissue engineering is a novel method wherein tissue is formed not in vitro previously, but in the patient's body. ${ }^{26}$

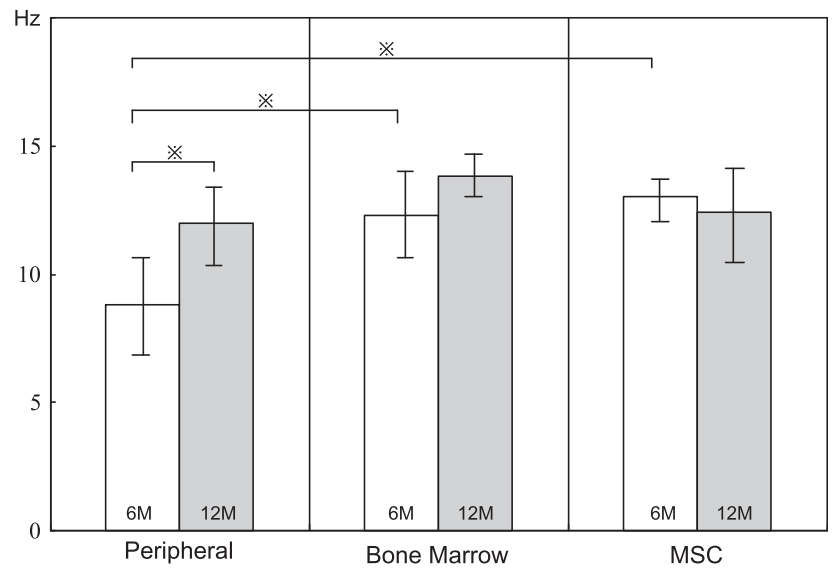

FIGURE 9. Cilial beat frequency of the regenerated cilia. MSC, Mesenchymal blood cell.
That is to say, in conventional tissue engineering, a required tissue has to be made previously on an incubation dish in the laboratory room. By contrast, in in situ tissue engineering, a scaffold of tissue is placed directly at the site of a tissue defect in the body where regeneration of the tissue is required.

In our early experiment, in which a prototype Y-shaped tracheal prosthesis was simply coated with amorphous collagen alone, 13 of 20 dogs died within 11 days after tracheal reconstruction. ${ }^{7}$ The main reason for these early postoperative deaths was air leakage from the trunk of the prosthesis. To address this problem, $\mathrm{we}^{27}$ improved the structure of the tracheal wall by increasing the amount of collagen through introduction of a porous collagen layer that was laid over the amorphous collagen layer. This improvement made the surgical procedure easier, and neither fibrin glue nor pledget sealing was necessary at the anastomosis sites during the operation.

For evaluation of functional recovery of the reconstructed tracheal wall, we measured the CBF of the regenerated

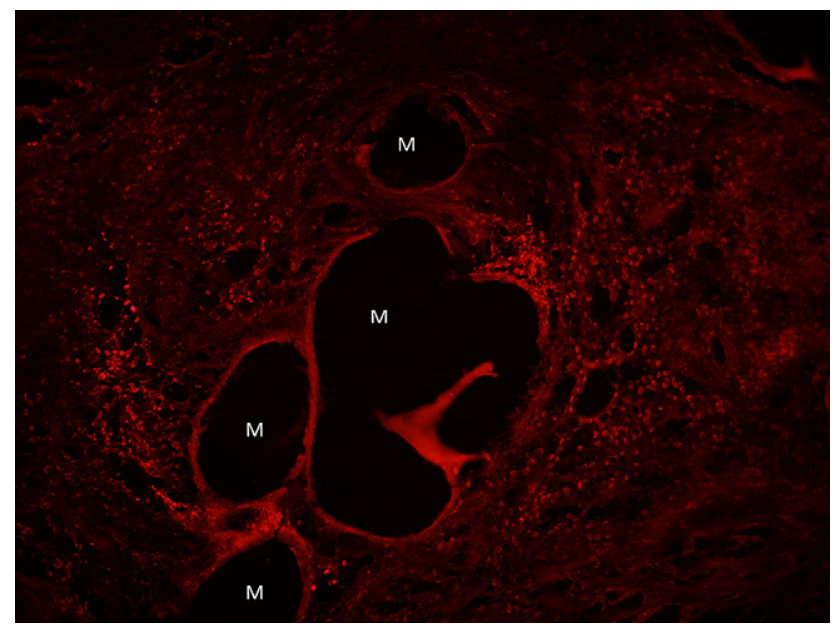

FIGURE 10. Microscopic appearance of the regenerated part of the tracheal wall after 1 month, showing numerous seeding MSCs, which are labeled with FM-DiI fluorescent tracer (original magnification, $\times 100$ ). The labeled cells remained in the submucosa tissue around the polypropylene mesh and did not exist in the mucosa. $M$, Mesh of the prosthesis). 
tracheal epithelium. Cilia play a major role in preserving the functional integrity of the airway, and CBF has been used as a marker of tracheal function in the field of pulmonology. ${ }^{28}$ The CBF value of the regenerated cilial area was $80 \%$ to $90 \%$ of the normal value in the bone marrow and MSC groups at 6 months and significantly higher than that in the peripheral blood group, although by 12 months the difference among the three groups was not significant. This may indicate that the reconstructed area had recovered not only morphologically but also functionally.

In our previous series of experiments, we $\mathrm{w}^{2-6}$ had soaked the collagen sponge of the prosthesis with peripheral blood during the operation. Because collagen has high affinity for platelets, ${ }^{29}$ we expected that the platelets attached to the collagen might provide platelet-derived growth factor or other active molecules that would help to enhance tissue regeneration. In the present study, in addition to peripheral blood used previously, we applied two other types of soaking medium: a bone marrow aspirate and a suspension of incubated bone marrow-derived cells. The latter cells have a tendency to attach to the bottom of the incubation bottle. Inasmuch as these cells have the potential to differentiate into several types of mesenchymal tissue, such as muscle, fat, and bone, they have been called multipotential bone marrowderived cells or MSCs. ${ }^{30}$ These cells have already been applied clinically as a cell source for tissue engineering. ${ }^{31}$

The results obtained in the bone marrow aspirate and MSC groups were better than those in the peripheral blood group. Mesh exposure and luminal stenosis occurred in only 2 dogs of the former two groups. These complications were milder in degree than those in the peripheral blood group. There was no significant difference in performance between the bone marrow aspirate group and the MSC group. In contrast, in the peripheral blood group, small areas of either mesh exposure or luminal stenosis occurred in all of the dogs but did not threaten the general condition of the animals or cause any significant problems. Evaluation of the functional recovery of the reconstructed site in terms of cilial movement revealed a similar tendency. Therefore, the application of bone marrow and MSCs to our prosthesis seemed to facilitate healing of the reconstructed tracheal region.

In the present study, we found that the implanted MSCs proliferated into the tracheal tissue. However it still remains unclear whether the cells in the soaked bone marrow aspirate also proliferated into the tracheal tissue. It will also be necessary to carry out further studies of the mechanism whereby soaked cells assist in tracheal remodeling on the collagen scaffold.

From a clinical viewpoint, use of a patient's own bone marrow aspirate seems to have many potential advantages. Aspiration of $2 \mathrm{~mL}$ of bone marrow from an anesthetized patient is relatively easy in the operating room and requires no incubation process to encourage cell proliferation. Eliminating the use of fetal bovine serum also obviates the risk of other unknown viral infections. In this regard, the application of MSCs in this experiment or iPS (induced pleuripotent) stem cells drawing current interest of the world have the same disadvantage; their clinical safety in terms of virus infection or tumorigenicity has not been established, inasmuch as fetal bovine serum or other nonautologous substances are now indispensable for preparation of such MSCs as well as iPS (induced pleuripotent) stem cells. ${ }^{32}$

In conclusion, although further long-term observation is required, our tracheal prosthesis appears promising for the repair of tracheal defects, and the application of autologous bone marrow may be clinically most feasible to assist the regeneration of tracheal tissue.

\section{References}

1. Grillo HC. Reconstruction of the trachea. Experience in 100 consecutive cases. Thorax. 1973;28:667-79.

2. Mulliken JB, Grillo HC. The limits of tracheal resection with primary anastomosis: further anatomical studies in man. J Thorac Cardiovasc Surg. 1968;55: 418-21.

3. Okumura N, Nakamura T, Natsume T, Tomihata K, Ikada Y, Shimizu Y. Experimental study on a new tracheal prosthesis made from collagen-conjugated mesh. J Thorac Cardiovasc Surg. 1994;108:337-45.

4. Teramachi M, Okumura N, Nakamura T, Yamamoto Y, Kiyotani T, Takimoto Y, et al. Intrathoracic tracheal reconstruction with a collagen-conjugated prosthesis: evaluation of the efficacy of omental wrapping. J Thorac Cardiovasc Surg. 1997; 113:701-11.

5. Sekine T, Nakamura T, Matsumoto K, Liu Y, Ueda H, Tamura N, et al. Carinal reconstruction with a Y-shaped collagen-conjugated prosthesis. J Thorac Cardiovasc Surg. 2000;119:1162-8.

6. Omori K, Nakamura T, Kanemaru S, Asato R, Yamashita M, Tanaka S, et al. Regenerative medicine of the trachea: the first human case. Ann Otol Rhinol Laryngol. 2005; 114:429-33.

7. Sekine T, Nakamura T, Liu Y, Ueda H, Matsumoto K, Shimizu Y. Collagen coated $\mathrm{Y}$-shaped prosthesis for carinal replacement promotes regeneration of the tracheal epithelium. ASAIO J. 2000;46:421-5.

8. Kawaguchi S, Nakamura T, Shimizu Y, Masuda T, Takigawa T, Liu Y, et al. Mechanical properties of artificial tracheas composed of a mesh cylinder and a spiral stent. Biomaterials. 2001;22:3085-90.

9. Lindberg S, Khan R, Runer T. The effects of formoterol, a long-acting beta 2-adrenoceptor agonist, on mucociliary activity. Eur J Pharmacol. 1995;285: 275-80.

10. Theman TE, Kerr JH, Nelems JM, Pearson FG. Carinal resection: a report of two cases and a description of the anesthetic technique. J Thorac Cardiovasc Surg. 1976;71:314-20.

11. Daniel RA. The regeneration of defects of the trachea and bronchi: an experimental study. J Thorac Surg. 1948;17:335-49.

12. Neville WE, Bolanowski JP, Kotia GG. Clinical experience with the silicone tracheal prosthesis. J Thorac Cardiovasc Surg. 1990;99:604-12; discussion 612-3.

13. Matsubara Y, Kosaba S, Ikeda S, Hanawa T, Shiota T, Ishida H, et al. [Experimental and clinical results of tracheal prosthesis]. Kyobu Geka. 1990;43:368-73; discussion 373-4.

14. Shimizu Y, Tamura K, Kato H, Teramatsu T, Hino T. Study of artificial trachea using mesh. Jpn J Artif Organs. 1983;12:486-9.

15. Pearson FG, Henderson RD, Gross AE, Ginsberg RJ, Stone RM. The reconstruction of circumferential tracheal defects with a porous prosthesis. an experimental and clinical study using heavy Marlex mesh. J Thorac Cardiovasc Surg. 1968;55: 605-16.

16. Poticha SM, Lewis FJ. Experimental replacement of the trachea. J Thorac Cardiovasc Surg. 1966;52:61-7.

17. Jacobs JR. Investigations into tracheal prosthetic reconstruction. Laryngoscope. 1988;98:1239-45

18. Ruoslahti E, Hayman EG, Pierschbacher MD. Extracellular matrices and cell adhesion. Arteriosclerosis. 1985;5:581-94.

19. Davenport EA, Nettesheim P. Regulation of mucociliary differentiation of rat tracheal epithelial cells by type I collagen gel substratum. Am J Respir Cell Mol Biol. 1996;14:19-26. 
20. Langer R, Vacanti JP. Tissue engineering. Science. 1993;260:920-6.

21. Sakata J, Vacanti CA, Schloo B, Healy GB, Langer R, Vacanti JP. Tracheal composites tissue engineered from chondrocytes, tracheal epithelial cells, and synthetic degradable scaffolding. Transplant Proc. 1994;26:3309-10.

22. Rainer C, Wechselberger G, Bauer T, Neumeister MW, Lille S, Mowlavi A, et al. Transplantation of tracheal epithelial cells onto a prefabricated capsule pouch with fibrin glue as a delivery vehicle. J Thorac Cardiovasc Surg. 2001;121: 1187-93.

23. Vacanti CA, Paige KT, Kim WS, Sakata J, Upton J, Vacanti JP. Experimental tracheal replacement using tissue-engineered cartilage. J Pediatr Surg. 1994;29: 201-4; discussion 204-5.

24. Kojima K, Ignotz RA, Kushibiki T, Tinsley KW, Tabata Y, Vacanti CA. Tissueengineered trachea from sheep marrow stromal cells with transforming growth factor beta2 released from biodegradable microspheres in a nude rat recipient. J Thorac Cardiovasc Surg. 2004;128:147-53.

25. Okamoto T, Yamamoto Y, Gotoh M, Huang CL, Nakamura T, Shimizu Y, et al. Slow release of bone morphogenetic protein 2 from a gelatin sponge to promote regeneration of tracheal cartilage in a canine model. J Thorac Cardiovasc Surg. 2004;127:329-34
26. Hori Y, Nakamura T, Kimura D, Kaino K, Kurokawa Y, Satomi S, et al. Experimental study on tissue engineering of the small intestine by mesenchymal stem cell seeding. J Surg Res. 2002;102:156-60.

27. Natsume T, Ike O, Okada T, Takimoto N, Shimizu Y, Ikada Y. Porous collagen sponge for esophageal replacement. J Biomed Mater Res. 1993;27:867-75.

28. Devalia JL, Sapsford RJ, Rusznak C, Toumbis MJ, Davies RJ. The effects of salmeterol and salbutamol on ciliary beat frequency of cultured human bronchial epithelial cells, in vitro. Pulm Pharmacol. 1992;5:257-63.

29. Roest M, Sixma JJ, Wu YP, Ijsseldijk MJ, Tempelman M, Slootweg PJ, et al. Platelet adhesion to collagen in healthy volunteers is influenced by variation of both $\alpha 2 \beta 1$ density and von Willebrand factor. Blood. 2000;96:1433-7.

30. Pittenger MF, Mackay AM, Beck SC, Jaiswal RK, Douglas R, Mosca JD, et al. Multilineage potential of adult human mesenchymal stem cells. Science. 1999; 284:143-7.

31. Adachi N, Ochi M, Deie M, Ito Y. Transplant of mesenchymal stem cells and hydroxyapatite ceramics to treat severe osteochondral damage after septic arthritis of the knee. $J$ Rheumatol. 2005;32:1615-8.

32. Rubio D, Garcia-Castro J, Martin MC, de la Fuente R, Cigudosa JC, Lloyd AC, et al. Spontaneous human adult stem cell transformation. Cancer Res. 2005;65:3035-9. 